\title{
ASOCIACIÓN ENTRE DIABETES MELLITUS Y CIRROSIS HEPÁTICA. JIPIJAPA. MANABI.ECUADOR
}

\author{
AUTORES: María Emilia Fernández Águilar ${ }^{1}$ \\ Ramón Palma Franco ${ }^{2}$ \\ Bárbara Miladys Placencia López ${ }^{3}$ \\ Holanda Mariola Merchán Ponce ${ }^{4}$ \\ Meriño Conforme Monserrate Cristina ${ }^{5}$
}

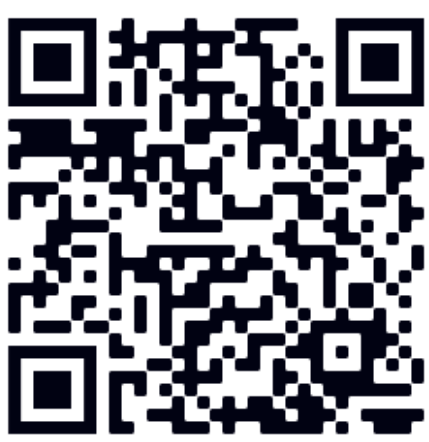

DIRECCIÓN PARA CORRESPONDENCIA:mariaefa602211@gmail.com

Fecha de recepción: 13/08/2019

Fecha de aceptación: 30/11/2019

\section{RESUMEN}

La presente investigación se desarrolló con el objetivo de revisar la asociación entre diabetes y cirrosis hepática en pacientes ecuatorianos. Se realizó un estudio descriptivo, longitudinal, retrospectivo. Se seleccionaron los 64 enfermos atendidos en consultas de gastroenterología entre enero 2015 y enero 2018, en el Hospital Ambulatorio del Seguro Social de Jipijapa, Manabí, Ecuador, con diagnóstico de cirrosis y diabetes mellitus, mayores de 20 años. Se revisaron las historias del Modelo AS 400 y se recolectaron los datos necesarios, analizándose causas etiológicas, diagnóstico y fecha de debut de la diabetes, fallecimiento o diagnóstico de hepatocarcinoma. Para el análisis estadístico se utilizó la tabla de frecuencia en el software SPSS versión 22.0 para Windows. La cirrosis hepática estuvo asociada a la diabetes en el $45 \%$ de los pacientes y fue más frecuente entre los de más de 60 años y del sexo masculino. Según el tiempo de diagnóstico, el 34,37\% tenía diagnóstico de diabetes antes de conocer que sufrían de cirrosis, mientras el 7,81\% debutaron diabéticos después de la cirrosis. La esteatohepatitis por diabetes mellitus asociada al alcoholismo, se observó como la causa más frecuente de cirrosis $(34,48 \%)$. El $13,79 \%$ presentó carcinoma hepatocelular y el $17,25 \%$ de los pacientes fallecieron.

PALABRAS CLAVE: enfermedad crónica hepática, fibrosis, intolerancia a la glucosa, insulino resistencia

\footnotetext{
${ }^{1}$ Especialista en gastroenterología. Master en longevidad satisfactoria. Gastroenteróloga Hospital Ambulatorio IESS de Jipijapa. Docente UNESUM. Jipijapa. Manabí. Ecuador mariaefa602211@ gmail.com

2 Especialista en gastroenterología. Gastroenterólogo. Hospital Ambulatorio IESS de Jipijapa. Manabí. Ecuador. ramonpalma2008@hotmail.com

${ }^{3}$ Especialista en Medicina Familiar. Médico especialista en Medicina Familiar Hospital Ambulatorio IESS de Jipijapa. Docente UNESUM. Jipijapa. Manabí. Ecuador. merchan.holanda@unesum.edu.ec

${ }^{4}$ Especialista en Medicina Familiar. Médico especialista en Medicina Familiar Hospital Ambulatorio IESS de Jipijapa. Docente UNESUM. Jipijapa. Manabí. Ecuador. merchan.holanda@ unesum.edu.ec

55 Licenciada en enfermería. Hospital Ambulatorio IESS de Jipijapa. Manabí. Ecuador. Master en gerencia en salud para el desarrollo local. Docente UNESUM. monserratemerinoconforme@ hotmail.com
} 
«Autor1», «Autor2», «Autor3»...

\section{ASSOCIATION BETWEEN DIABETES MELLITUS AND HEPATIC CIRRHOSIS, JIPIJAPA, MANABÍ, ECUADOR}

\section{ABSTRACT}

The present investigation was developed with the objective of reviewing the association between diabetes and liver cirrhosis in Ecuadorian patients. A descriptive, longitudinal, retrospective study was carried out. The 64 patients treated at gastroenterology clinics between January 2015 and January 2018 were selected at the Outpatient Hospital of the Social Security of Jipijapa, Manabí, Ecuador, diagnosed with cirrhosis and diabetes mellitus, over 20 years of age. The histories of the Model AS 400 were reviewed and the necessary data were collected, analyzing etiological causes, diagnosis and date of debut of the diabetes, death or diagnosis of hepatocarcinoma. For the statistical analysis, the frequency table was used in the software SPSS version 22.0 for Windows. Liver cirrhosis was associated with diabetes in $45 \%$ of patients and was more frequent among those over 60 years of age and male. According to the time of diagnosis, $34.37 \%$ had a diagnosis of diabetes before knowing that they suffered from cirrhosis, while $7.81 \%$ had diabetics after cirrhosis. Steatohepatitis due to diabetes mellitus associated with alcoholism was observed as the most frequent cause of cirrhosis (34.48\%). 13.79\% presented hepatocellular carcinoma and 17.25\% of the patients died.

KEYWORDS: chronic liver disease, fibrosis, glucose intolerance, insulin resistance

\section{INTRODUCCIÓN}

La cirrosis hepática $(\mathrm{CH})$ ocupa el séptimo lugar como causa de muerte en el Ecuador con 2323 defunciones, con tasa anual de mortalidad de $6,72 \%$ por cada 10000 habitantes según informes del Instituto Nacional de Estadísticas y Censo del Ecuador en su Anuario de estadísticas vitales nacimientos y defunciones 2016 (INEC 2017, p. 43-46). Mientras la diabetes mellitus (DM) afecta a la población con tasas cada vez más elevadas. La encuesta (ENSANUT, 2011-2013) reporta, la prevalencia de diabetes en la población de 10 a 59 años es de 1.7\%. Esa proporción se incrementa a partir de los 30 años de edad, y a los 50, uno de cada diez ecuatorianos ya tiene diabetes. La mortalidad por esta patología fue de 4906 defunciones en el 2016, con una tasa anual de mortalidad de 14,73\% por cada 10000 habitantes, solo superada por la enfermedad isquémica del corazón (INEC 2017, p. 43-46).

La asociación entre diabetes mellitus de tipo 2 hereditaria y cirrosis hepática ha sido documentada a través del tiempo, sobre todo cuando se asocia al síndrome metabólico, obesidad e hipertrigliceridemia, (García-Compeán, 2013 p. 474) pero también se conoce que la diabetes puede ser una complicación de la cirrosis, denominándose como "diabetes hepatógena". Ambas se asocian con un aumento de las complicaciones y muerte en enfermos con cirrosis.

En la actualidad se registra en todo el mundo un incremento de la esteatohepatitis no alcohólica (EHGNA) como causa de $\mathrm{CH}$, entidad indisolublemente ligada a la diabetes, hipertrigliceridemia y obesidad. La incidencia de la EHGNA ha aumentado considerablemente en paralelo al alarmante crecimiento de la obesidad y el sobrepeso en países desarrollados y en numerosos países emergentes, situación también presente en Ecuador.

Estudios realizados en el 2018 de "Causas de cirrosis hepática en pacientes del hospital del seguro social de Jipijapa Manabí”, demostraron que la EHGNA supera en estos momentos al alcohol y los 
virus como factores etiológicos, aunque en muchos pacientes estos factores se asocian. (Fernández at al, 2018).

El tratamiento de la DM del paciente cirrótico es difícil por lo siguiente: $a$ ) alrededor de la mitad de los pacientes tiene malnutrición; $b$ ) cuando se diagnostica la DM el paciente tiene insuficiencia hepática avanzada; $c$ ) la mayor parte de los hipoglucemiantes orales y la insulina se metabolizan en el hígado; $d$ ) estos pacientes tienen con frecuencia episodios de hipoglucemia; $e$ ) la adherencia al tratamiento posiblemente es baja, particularmente en los alcohólicos, y $f$ ) la persistencia de la ingestión de alcohol (Tolman, 2007, p. 736).

En el trabajo cotidiano en las consultas de gastroenterología en el Hospital Ambulatorio del Seguro Social de Jipijapa, Manabí, se maneja esta problemática por lo que nos motivamos a desarrollar la presente investigación con el objetivo de revisar la asociación entre diabetes y cirrosis hepática en pacientes ecuatorianos.

\section{DESARROLLO}

\section{MATERIALES Y METODOS}

Se realizó un estudio descriptivo, longitudinal, retrospectivo, con pacientes atendidos en consulta de gastroenterología, en el Hospital Ambulatorio del Día, IESS Jipijapa. Manabí, Ecuador, con el objetivo de determinar la asociación entre diabetes mellitus y cirrosis hepática, en el período del 1 de enero de 2015 al 1 de enero de 2018. El universo de estudio estuvo formado por 64 pacientes que tenían diagnostico confirmado de $\mathrm{CH}$. Se seleccionaron aquellos que cumplían los criterios de inclusión (diagnóstico confirmado de cirrosis hepática, edad igual o mayor a 20 años, historia clínica en la que se constató la existencia de diabetes mellitus, o hipertrigliceridemia (dislipemia) confirmada por datos clínicos y de laboratorio, obesidad determinada por el índice de masa corporal, diagnóstico confirmado de hepatocarcinoma, fecha de fallecimiento .

Se revisaron las historias clínicas del modelo AS 400, para obtener información acerca de edad, sexo, fecha del diagnóstico de la enfermedad. Se definió cirrosis por la suma de criterios clínicos, datos de laboratorio, endoscópicos, métodos imagenológicos o mediante la demostración histológica. Se revisó el diagnostico confirmado de diabetes mellitus tipo II en la historia clínica por las cifras elevadas de glicemia en ayuna, o de hemoglobina glicosilada, historia previa de diabetes o de tratamiento de la misma. Se definió "diabetes hepatógena", cuando el diagnóstico de diabetes fue después de diagnosticada la $\mathrm{CH}$. Además se analizó cuando el debut de ambas enfermedades coincidió. Se analizaron posibles causas etiológicas, para lo que se operacionalizaron las diferentes variables. Se definió la ingestión de alcohol como causa de CH, si el paciente tenía el antecedente de alcoholismo o era un bebedor de riesgo según clasificación de Marconi (Del Sol, 2010, p. 61). El diagnóstico CH por hepatitis viral se estableció por pruebas de serología positiva para hepatitis B o C (ELISA tercera generación), realizadas en el laboratorio del IESS. En los enfermos en que no se encontró etiología de cirrosis pero tenían antecedentes de obesidad mórbida, diabetes mellitus o dislipemia (hipertrigliceridemia), se consideró como causa probable de $\mathrm{CH}$ la esteatosis hepática no alcohólica. Se revisaron otras causas, como autoinmune, Wilson, hemocromatosis, teniendo en cuenta los criterios internacionales utilizados en el diagnóstico de las mismas. Solo se consideraron complicaciones como el diagnóstico de hepatocarcinoma $(\mathrm{CHC})$ o fallecimiento en estos enfermos. De dicha revisión se obtuvo toda la información necesaria para desarrollar el modelo de vaciamiento de datos, lo que constituyó la fuente fundamental de la investigación. 
«Autor1», «Autor2», «Autor3»...

El análisis estadístico se realizó a través del uso de la estadística descriptiva, para lo que se determinaron las frecuencias por rango de edades y enfermedades, se desarrolló el estadístico de contraste observado que sigue aproximadamente un Chi-cuadrado con un nivel de significación $\alpha=0,05$ y resultó la frecuencia esperada mayor que $\mathrm{x}^{2}$, por lo que se acepta la hipótesis nula y en consecuencia la variable aleatoria edad de los pacientes con Diabetes y $\mathrm{CH}$.

Para entender con mejor precisión el criterio acerca de la edad y frecuencia de enfermos de Diabetes + C.H, se analizó las frecuencias de respuesta a través de un diseño de bloques completamente aleatorizados, donde se bloqueó el efecto de la edad y se realizaron ocho repeticiones. Previo al análisis de varianza, se determinó la distribución de normalidad y homogeneidad de varianzas. Si las medias de frecuencia no se ajustasen a una curva normal se transformaron mediante raíz cuadrada $(\sqrt{ }(\mathrm{X}+0.5)$ para normalizar los datos de frecuencia y realizar la prueba de Tukey (HSD) para frecuencias y determinar si existen diferencias entre las edades de los enfermos por sexos.

No se entrevistaron a los pacientes para obtener la información ya que los datos de las encuestas se obtuvieron directamente del modelo AS 400. Quedó bien explícita la necesidad de guardar absoluta discreción en el manejo de los mismos según los principios bioéticos relacionados con las investigaciones en seres humanos de la declaración de Helsinki. Se pidió autorización al Comité de Ética Médica de la Institución para realizar la investigación, no existen conflictos de intereses.

\section{RESULTADOS Y DISCUSIÓN}

El 45\% de los pacientes con cirrosis hepática presentaba diabetes mellitus, como se aprecia en la figura 1. García Compeán, en un estudio realizado en el Hospital Universitario de Monterrey, México, en el 2012, en 130 pacientes con $\mathrm{CH}$ observó que el 27,6\% tenía diabetes manifiesta, y el $10,7 \%$ intolerancia a la glucosa.

Trombeta (2005, p. 23) por su parte reportó la prevalencia de diabetes en la cirrosis entre el 12,3 - 57\%, en un estudio realizado en Verona en el 2005 en 7148 pacientes diabéticos tipo 2 después de 5 años de seguimiento y Kobashi $(2010$, p. 283) en "Prevalencia de diabetes mellitus tipo 2 y enfermedad hepática crónica: un estudio retrospectivo de la asociación de dos enfermedades cada vez más comunes en México" relacionó significativamente la cirrosis hepática con la diabetes mellitus tipo 2 (21 de 61 pacientes; 34,4\%).

La asociación entre DM y $\mathrm{CH}$ con frecuencia ha sido subestimada por los médicos, pero estudios de la última década demuestran que EHNA hoy es causa de cirrosis hepática en un número creciente de pacientes y es claro su vínculo con la diabetes, obesidad, e hipertrigliceridemia, evento que se denomina "La nueva pandemia del milenio" (Santos L. et al, 2010, p. 380). 


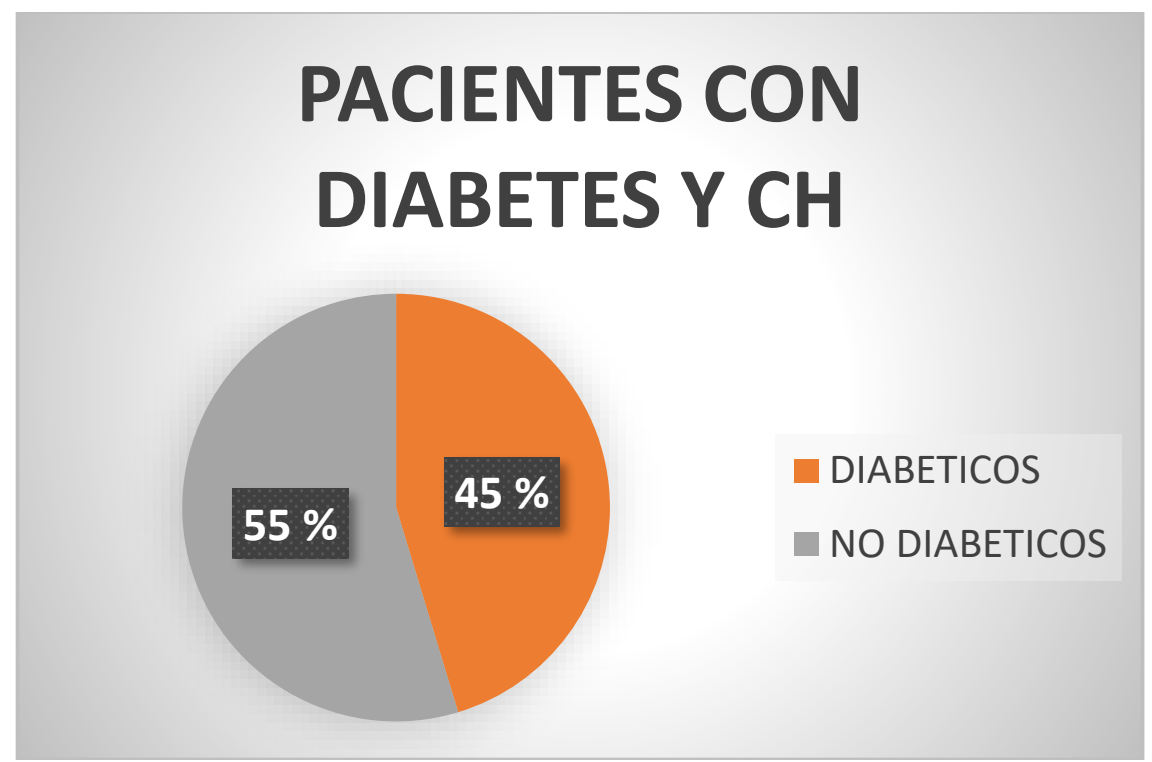

Figura 1. Relación de pacientes con cirrosis hepática y diabetes

La figura 2 muestra la distribución de pacientes enfermos por cirrosis hepática y diabetes según grupos de edad y sexo. Se observa que el diagnóstico de diabetes y cirrosis aumenta con la edad, correspondiendo al grupo de pacientes con edades iguales o mayores a 60 años el 29,68\%. Respecto al sexo, en el masculino esta asociación fue del 28,15\% superior a la que se observó en las mujeres.

Los resultados respecto a la edad, difieren a los reportes de Hernández Ortega, et al. (2014, p. 269) y Moreno T, (2015, p. 37) que muestran asociación entre DBT y CH en un 37,04\% en mayores de 60 años y $46 \%$ respectivamente. Estos autores reportan también mayor incidencia en el sexo masculino, con lo que coincide la presente investigación, pero en cifras superiores $(77,8 \%$ y $62 \%)$. Sin embargo Kobashi (2010, p. 283) en México reporta mayor incidencia de esta asociación por encima de los 56 años con una media de 16 años, en el 30\% de los hombres, con lo que concuerda este estudio.

La mayoría de los estudios sobre $\mathrm{CH}$ coinciden en que la edad de presentación más frecuente es la de más de 60 años, pues los factores (alcohol, virus, EHNA, entre otros) que desencadenan la enfermedad ya actuaron a través del tiempo, lo que determinó los cambios anatomo-patológicos típicos de esta afección, que muchas veces permanece asintomática hasta estas edades en que debuta con complicaciones. Por otro lado la prevalencia de diabetes en estas edades, no ha dejado de incrementarse en las últimas décadas; esto acontece como consecuencia de una serie de factores, entre los que deben mencionarse la mayor edad de la población, el progresivo incremento de la obesidad y el sedentarismo entre muchos grupos sociales, motivados por cambios en sus hábitos de vida, situación de la que no escapa la población estudiada.

Se considera que el aumento de la longevidad en la población general, el mejor control de las hepatitis virales $\mathrm{B}$ y $\mathrm{C}$, los hábitos de vida que incluyen la alimentación y el sedentarismo, explicarían los resultados que se mostraron.

Las frecuencias por rango de edades y número de pacientes se muestran en la tabla 1.

Tabla 1. Análisis de frecuencias por rango de edades y número de pacientes 
$\ll$ Autor $1 », \ll$ Autor $2 », \ll$ Autor $3 » \ldots$

\begin{tabular}{|l|l|l|l|}
\hline Edad (clase) & $\mathbf{X}_{\mathbf{1}}$ & $\begin{array}{l}\text { Pacientes } \\
\text { (frecuencia) }\end{array}$ & Porciento \% \\
\hline$(40-49)$ & 44,5 & 1 & 3,5 \\
\hline$(50-59)$ & 54,5 & 9 & 31 \\
\hline$(60-69)$ & 64,5 & 19 & 65,5 \\
\hline Total & & 29 & \\
\hline
\end{tabular}

El promedio de edad de los pacientes es de 45,48 años demostrando el estadístico de contraste, el que sigue aproximadamente un Chi-cuadrado con un nivel de significación $\alpha=0,05$ y resultó la frecuencia esperada $e=7,06>\mathrm{x}^{2}=5,2$ por lo que se acepta la hipótesis nula y en consecuencia la variable aleatoria edad de los pacientes con Diabetes y C.H.(figura 2)

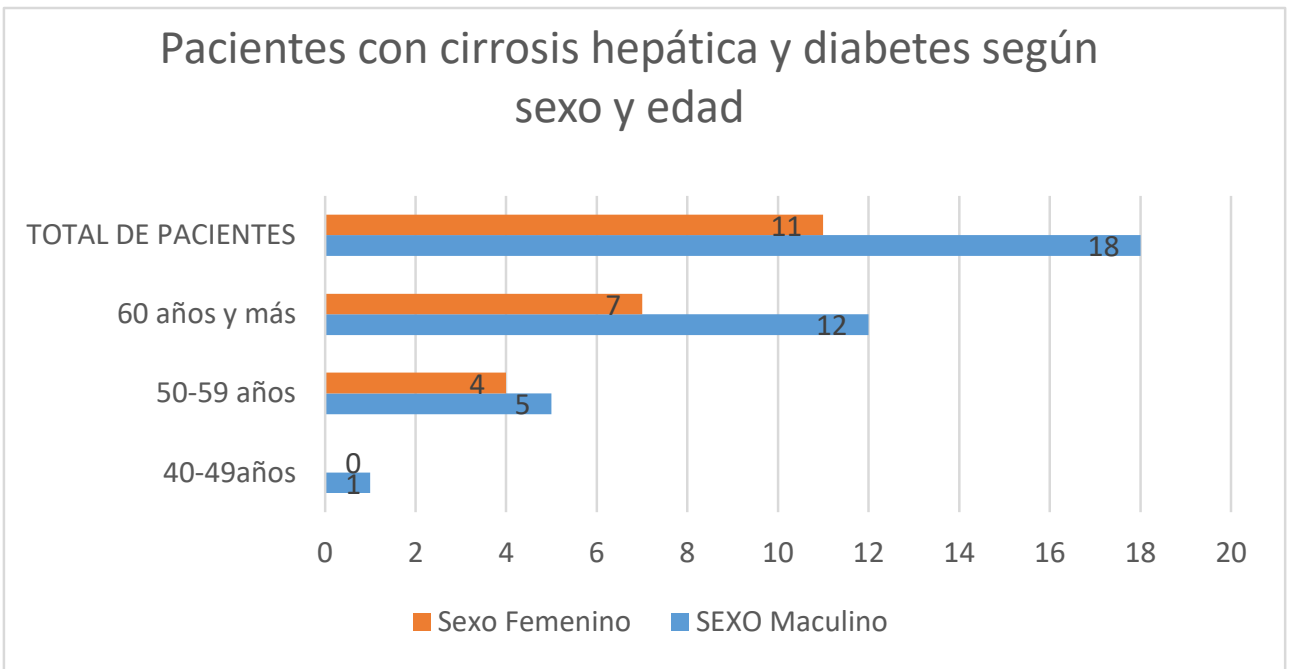

Figura 2. Distribución de pacientes afectados por Cirrosis Hepática y Diabetes según grupos de edad y sexo

En cuanto al momento de la realización del diagnóstico de la diabetes en relación al de la cirrosis (como se muestra en la figura 3), se encontró que en el 89,1\% la diabetes antecedió el diagnóstico de cirrosis, en el $7,8 \%$ este se realizó después y en el 3,1\% en forma simultánea.

En México, García Compeán et al (2012, p. 443), realizó el diagnóstico de diabetes antes de cirrosis en el $64 \%$, de los enfermos, después de la cirrosis en el $16 \%$, y en forma simultánea en el $20 \%$, con una amplia predominancia de pacientes que ya conocían el diagnóstico de diabetes antes que el de la cirrosis, con lo que coinciden estos hallazgos. Por su parte Hernández Ortega en Cuba reporta en el 22,2\% de los cirróticos diagnósticos de diabetes en la evolución de su enfermedad. 
El desarrollo de la diabetes en pacientes con cirrosis es bien conocido, pero está surgiendo evidencia de que el desarrollo de la enfermedad hepática crónica y la progresión a la cirrosis pueden ocurrir después del diagnóstico de diabetes y que la diabetes desempeña un papel en el inicio y la progresión de la lesión hepática (Ingrid, 2007, p 829), (García- Compeán et al, 2013, p 443). La diabetes hepatógena no es reconocida por la Asociación Americana de diabetes en su nueva clasificación, a pesar de que se reconoce esta como un parámetro de enfermedad hepática avanzada y que se asocia a un aumento de las complicaciones hepáticas, resistencia a los antivirales y muerte en los pacientes cirróticos.

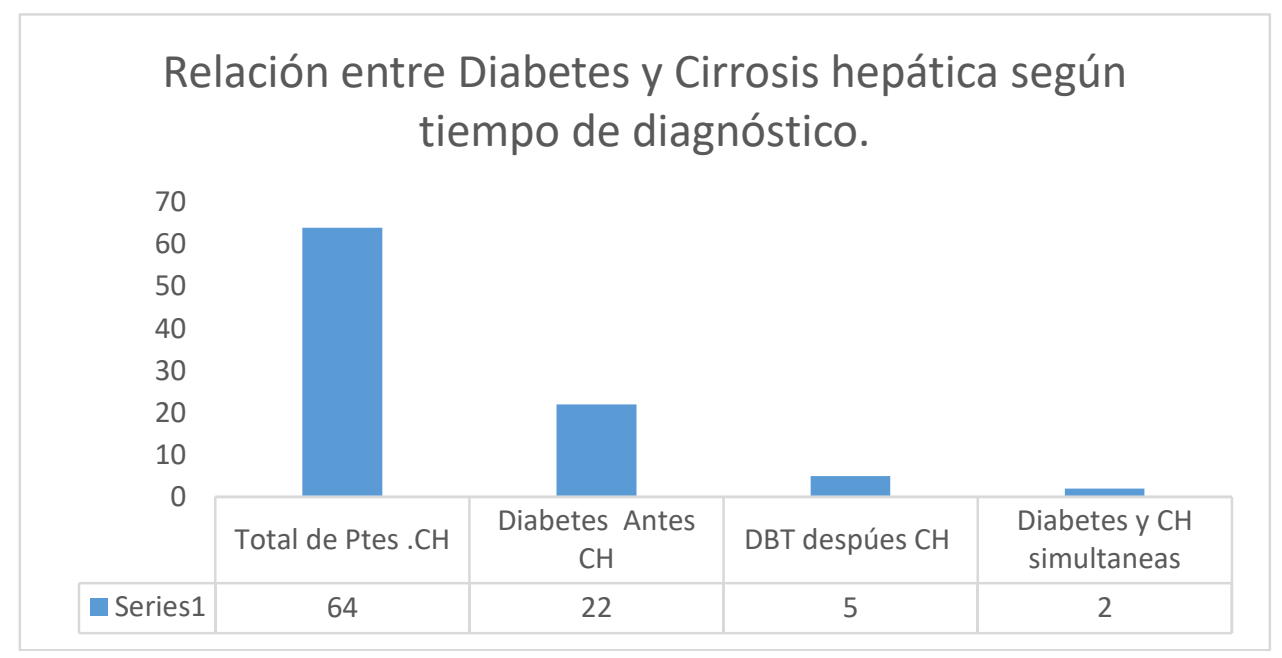

Figura 3. Relación entre Diabetes y Cirrosis hepática según tiempo de diagnóstico

Respecto a la etiología de la Cirrosis Hepática en los pacientes con diabetes y cirrosis, (como se observa en la tabla 2) se encontró que el alcoholismo asociado a la diabetes ocupaba el primer lugar con $34,48 \%$, con franco predomino en el sexo masculino. Entre las féminas la diabetes sola o asociada a la dislipemia o a la dislipemia y obesidad, como causa de EHNA se comportó de forma similar con $10,34 \%$ respectivamente.

La asociación de más de una causa etiológica también se observa en la tabla 2 y corrobora como se superponen el daño hepático causado por el alcohol, al daño causado por la diabetes, obesidad o dislipemia, esta asociación se evidenció en el 83\% de los 18 pacientes masculinos. Sin embargo el alcoholismo no estuvo presente en la población femenina lo que se considera guarde relación con la cultura y tradiciones de la región donde dicho hábito estigmatiza a la mujer y realmente no se reportó en estos grupos de edades entre las féminas.

Moreno T. (2015) en su estudio de Diabetes mellitus en la cirrosis hepática reporta el alcohol en la etiología del 8,62\% de los pacientes, cifra que asciende al $23 \%$ cuando combina los virus y el alcohol, en el $15 \%$ reporta causas no filiadas.

Hernández Ortega (2014) reporta en Cuba el 51,85\% de los pacientes presentaron cirrosis de causa alcohólica, el 25,92\% de causa viral. Por su parte Jáquez en el 2011 en "El impacto de la diabetes mellitus en la mortalidad de pacientes con cirrosis hepática compensada: un estudio prospectivo" informa el alcoholismo como causa de $\mathrm{CH}$ en el 40,9\% seguido de $\mathrm{CH}$ criptogenética 38,1\%. 
(Wei M. 2000, p 18) citado por García-Compeán (2013, p 476) menciona “Los pacientes con enfermedad hepática alcohólica tienen un riesgo relativo elevado de tener diabetes mellitus, el cual está en relación directa con la cantidad de alcohol ingerido ya que se aumenta al doble en los que ingieren más de $270 \mathrm{~g}$ de alcohol por semana en comparación con los que ingieren de 60 a 120 g”. Por otra parte, los pacientes con alcoholismo crónico con frecuencia presentan daño pancreático crónico y lesión de las células beta de los islotes pancreáticos, lo que produce como consecuencia DM".

Tabla 2. Etiología de la cirrosis en pacientes con asociación de cirrosis y diabetes.

\begin{tabular}{|c|c|c|c|c|c|c|}
\hline \multirow{3}{*}{ Etiología } & \multicolumn{4}{|c|}{ Sexo } & \multirow{2}{*}{\multicolumn{2}{|c|}{ Total }} \\
\hline & \multicolumn{2}{|c|}{ Masculino } & \multicolumn{2}{|c|}{ Femenino } & & \\
\hline & $\begin{array}{c}\mathrm{n}= \\
18\end{array}$ & $\%$ & $\begin{array}{l}\mathrm{n}= \\
11\end{array}$ & $\%$ & $29^{n=}$ & $\%$ \\
\hline Alcoholismo + Diabetes & 10 & 34,48 & 0 & 0,00 & 10 & 34,48 \\
\hline Alcoholismo + Diabetes + Hepatitis B & 1 & 3,44 & 0 & 0,00 & 1 & 3,44 \\
\hline $\begin{array}{l}\text { Alcoholismo }+ \\
\text { Diabetes }\end{array}$ & 1 & 3,44 & 0 & 0,00 & 1 & 3,44 \\
\hline $\begin{array}{l}\text { Alcoholismo }+ \text { Obesidad }+ \text { DBT }+ \\
\text { Dislipemia }\end{array}$ & 3 & 10,34 & 0 & 0,00 & 3 & 10,34 \\
\hline Dislipemia + Diabetes & 0 & 0,00 & 3 & 10,34 & 3 & 10,34 \\
\hline Obesidad + Diabetes + Dislipemia & 0 & 0,00 & 3 & 10,34 & 3 & 10,34 \\
\hline Obesidad + Diabetes & 1 & 3,44 & 2 & 6,89 & 3 & 10,34 \\
\hline Diabetes & 2 & 6,89 & 3 & 10,34 & 5 & 17.24 \\
\hline
\end{tabular}

Tratamientos, enfermedades $y$ frecuencias registradas en la prueba Prueba del rango estudentizado de Tukey (HSD) para Frecuencias. 


\begin{tabular}{|c|c|c|c|c|}
\hline coste & Font & 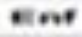 & 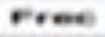 & Niven 7 \\
\hline 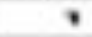 & 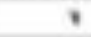 & r & 80 & 3. 200032 \\
\hline 2 & , & 2 & 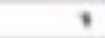 & B. D Dhet/4 \\
\hline $\boldsymbol{n}$ & , & 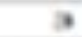 & , & V.D. \\
\hline 4 & , & 4 & 3 & 1. as:0us \\
\hline $\mathbf{5}$ & , & 6 & $\sigma$ & $0.700,1$ \\
\hline 6 & ? & 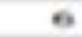 & 0 & $0,50 \%: 1$ \\
\hline 7 & 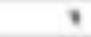 & 7 & , & 1.201474 \\
\hline a & , & A & 2 & 1. 560,74 \\
\hline $\mathbf{s}$ & 2 & , & 10 & D. 20007 \\
\hline 16 & 2 & 2 & , & 1.20147a \\
\hline 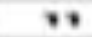 & 2 & 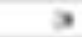 & $\checkmark$ & B. 2014,74 \\
\hline 12 & 2 & 4 & 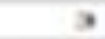 & 1. 6xon. \\
\hline 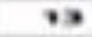 & 2 & 6 & 3 & 1. $6 \mathrm{x}, 20 \mathrm{cos}$ \\
\hline 10 & 2 & 6 & 3 & 1.6.700 \\
\hline 15 & 2 & 7 & $\rightarrow$ & 3, nxom \\
\hline 16 & 2 & 6 & 6 & 2.3 .4007 \\
\hline
\end{tabular}

Procedimiento GLM

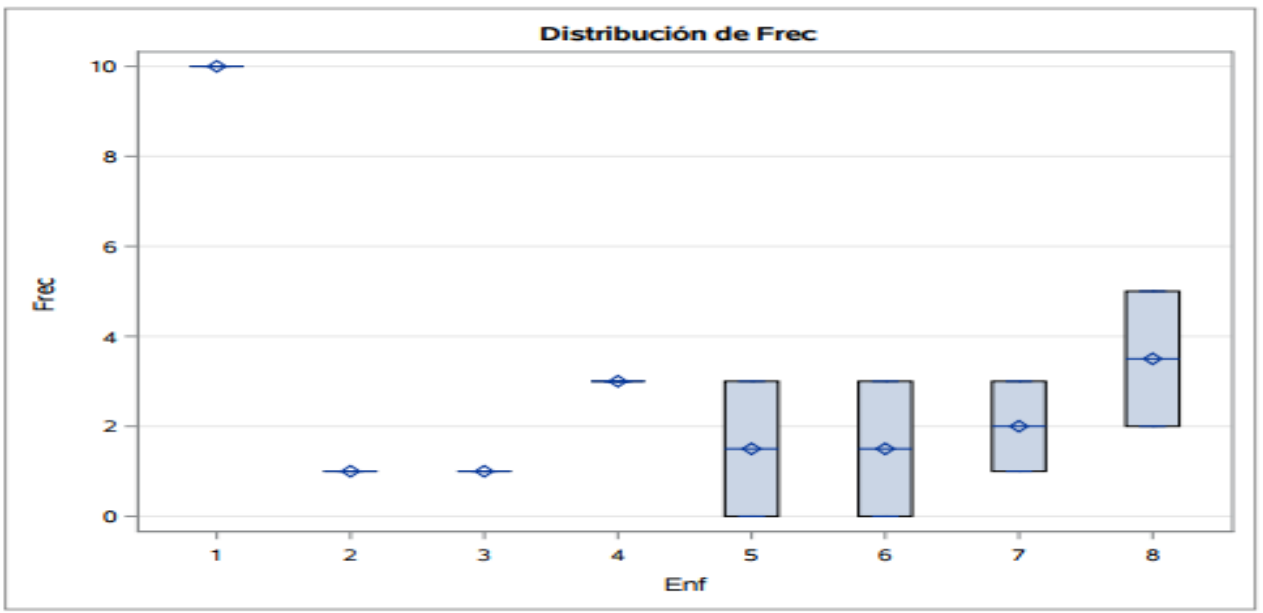

Procedimiento GLM Prueba del rango estudentizado de Tukey (HSD) para Frec1. 
«Autor1», «Autor2», «Autor3»...

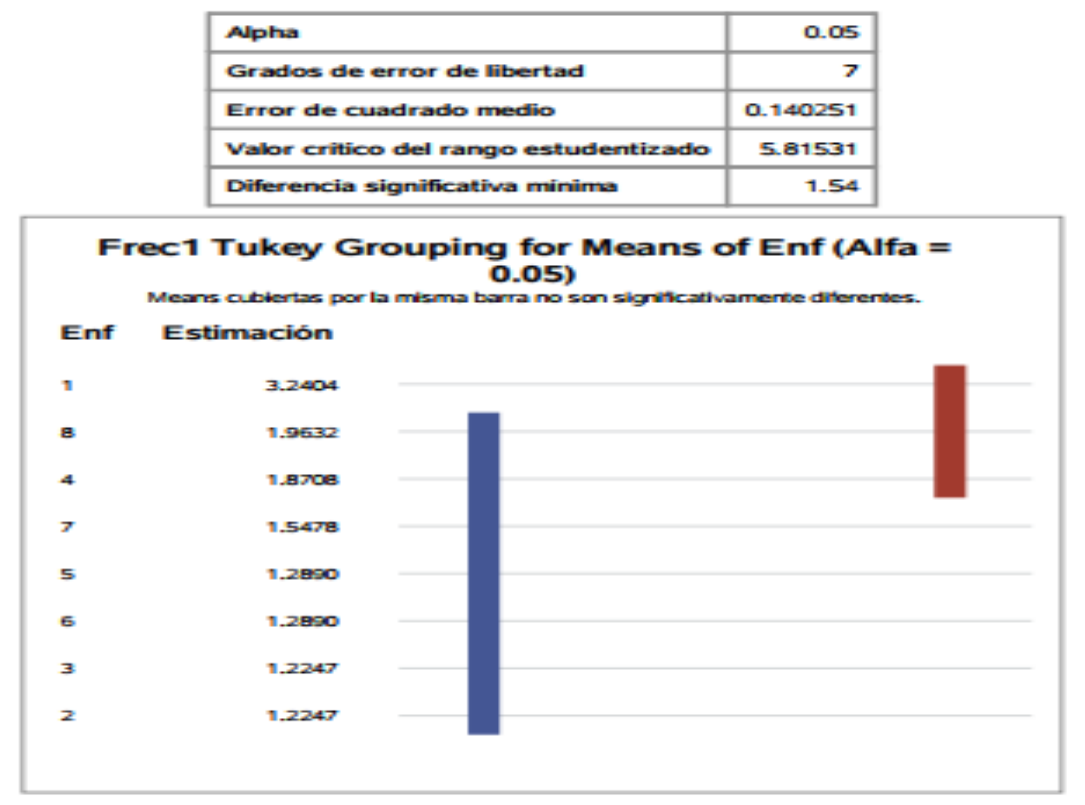

La prueba Tukey realizada con ocho tratamientos masculinos y ocho femeninos, teniendo en cuenta las frecuencias y las enfermedades demuestra que no existen diferencias significativas entre los hombres y las mujeres enfermos de diabetes y C.H

En 4 pacientes con cirrosis y diabetes se diagnosticó hepatocarcinoma y 5 fallecen por complicaciones de la cirrosis como se muestra en la tabla \# 2. De los fallecidos 4 tenían diagnóstico confirmado de carcinoma hepatocelular.

La diabetes mellitus aumenta el riesgo de desarrollar hepatocarcinoma, sobre todo si se presenta asociada a hepatitis, B, C o consumo de alcohol, situación que se dio en uno de los pacientes. El alcoholismo estuvo presente en 3 de los enfermos con CHC.

Kobashi reporta carcinoma hepatocelular en el $21 \%$ de los pacientes con asociación de cirrosis y diabetes, mientras Jázquez et al, 2011, reportan CHC en el 17\% de los pacientes con estas asociaciones. Por otra parte, en el estudio de Verona en el 2005 en 7148 pacientes diabéticos tipo 2 en la misma población después de 10 años de seguimiento, se observó un mayor riesgo de mortalidad por cáncer de hígado y este riesgo aumentó significativamente en pacientes obeso. (Índice de masa corporal $>30 \mathrm{~kg} / \mathrm{m}^{2}$ ) (Trombeta, 2005)

Los resultados de la presente investigación coinciden con García Compeán (2013, p. 478) cuando planteó "El aspecto más importante de la asociación de la cirrosis hepática con la DM o la DH radica en que la presencia de cualquiera de estos tipos de diabetes incrementa la morbilidad y la mortalidad. Por una parte la diabetes mellitus tipo 2 acelera la aparición de hepatocarcinoma en pacientes con cirrosis hepática y su deficiente control glucémico se ha asociado con incremento de la incidencia de complicaciones cardiovasculares y hepáticas así como de la mortalidad. Por otro lado la presencia de diabetes hepatógena acelera el proceso de fibrosis. Esto conduce a un agravamiento de la insuficiencia hepática y a la inducción de complicaciones y muerte derivadas de la enfermedad hepática (hemorragia por varices esofágicas, encefalopatía hepática, peritonitis 
bacteriana espontánea y síndrome hepatorrenal). Las complicaciones hepáticas son más frecuentes que las cardiovasculares con este tipo de diabetes (tabla 3).

Tabla 3. Distribución de pacientes según complicaciones de la cirrosis hepática y sexo

\begin{tabular}{lllll}
\hline & \multicolumn{4}{l}{ Total de pacientes con CH y diabetes } \\
& \multicolumn{2}{l}{ (n 29) } & \\
\cline { 2 - 5 } Complicaciones asociadas & \multicolumn{2}{l}{ Masculino } & \multicolumn{2}{l}{ Femenino } \\
\cline { 2 - 5 } & 4 & 13,79 & 0 & 0,00 \\
\hline Carcinoma Hepatocelular & 5 & 17,24 & 0 & 0,00
\end{tabular}

\section{CONCLUSIONES}

Se concluye que la cirrosis hepática estuvo asociada a la diabetes en el $45 \%$ de los pacientes y fue más frecuente entre los del sexo masculino con más de 60 años, mientras que el 34,37\% de los que padecían cirrosis eran diabéticos antes a su diagnóstico y el 7,81\% debutaron diabéticos después de la cirrosis. La esteatohepatitis por diabetes mellitus asociada al alcoholismo fue la causa más frecuente de cirrosis $(34,48 \%)$, seguidas de la asociación diabetes, alcoholismo, obesidad y dislipemia (10,34\%). El 13,79\% presentó carcinoma hepatocelular y el 17,25\% de los pacientes fallecieron.

\section{REFERÉNCIAS BIBLIOGRÁFICAS}

Del Sol Padrón, L. G., Fernández, O. Á., \& Berovides, J. D. D. R. (2010). Consumo de alcohol. Revista Finlay, 61-65. Recuperado:

www.revfinlay.sld.cu/index.php/finlayarticle/dowload/11/13de lgdesolpadron. 2010

Encuesta nacional de salud y nutrición del Ecuador ENSANUT-ECU). (2011-2013). del Ministerio de

Salud Pública. Recuperado de. http:// www.ecuadorencifras.gob.ec

Fernández Aguilar ME, Toala Bozada G., Placencia López BM., Merchán Ponce MH., Aliatis Bravo A. social de

(2018). "Causas de cirrosis hepática en pacientes del hospital ambulatorio del seguro

Jipijapa Manabí”, UNESUM-CIENCIAS, Vol.2 2018, No 2 p 61-71.ISSN 2602-8166 
«Autor1», «Autor2», «Autor3»...

García-Compeán D, Jáquez-Quintana J, González-González J, Lavalle-González F, VillarrealPérez J, hepatología.

Maldonado-Garza H (2013). La diabetes en la cirrosis hepática. Gastroenterología y

Vol. 36. (7).Ag - p.443-98 DOI: 10.1016/j.gastrohep.2013.01.012

García-Compeán D, Jáquez-Quintana J, González-González J, Lavalle-González F, ReyesCabello E,

González-González JA, Muñoz-Espinosa LE, Vázquez-Elizondo G, Villarreal-Pérez JZ, metabolism

Maldonado-Garza HJ. (2012) The prevalence and clinical characteristics of glucose Recuperado

disorders in patients with liver cirrhosis: A prospective study. Ann Hepatol; 11: 240-48

de: http://www.annalsofhepatology. com/revista/numeros/2012

Hernández Ortega A, Sánchez Cruz JC, Pereira Martínez MM, Umpierrez García I. (2014) Diabetes

Monroy.

mellitus como complicación de la cirrosis hepática. Hospital Docente Mario Muñoz

2003-2013. Rev. Méd. Electrón [Internet]. May. Jun. [citado: fecha de acceso]; 36 (3). Disponible

en: http://www.revmatanzas.sld.cu

Ingrid J. Hickman, Graeme A. Macdonald. (2007). Impacto de la diabetes en la gravedad de la enfermedad hepática. American Jornal of medicine. Vol. 120. (10). P 829-34 https://doi.org/10.1016/ j.amjmed.2007.03.025

Instituto Nacional de Estadística y Censos (INEC) (2017). Anuario de Estadísticas Vitales Nacimientos $y$

Defunciones. Recuperado http://www.ecuadorencifras.gob.ec/documentos/web/Nacimientos_Defunciones/2017/Presentacion_Nac_y_Def_2017.pdf

Jaquéz Quintana J., García-Compeán D, González-González J, Villarreal-Pérez JZ, LavalleGonzález F,

mellitus

Muñoz-Espinosa LE, López Hernández P, Pérez Cabello. (2011). The impact of diabetes hepatol. Jan -

in mortality of patients with compensated liver cirrhosis a prospective study. Ann march;10: Recuperado de: http://www.medigraphic.com/cgiin/new/resumen.cgi?IDARTICULO

Kobashi-Margáin R., Gutiérrez-Grobe Y. , Ponciano-Rodríguez G., Uribe M. , Méndez-Sánchez N. (2010). 
Prevalencia de diabetes mellitus tipo 2 y enfermedad hepática crónica: un estudio retrospectivo de sep; 9

la asociación de dos enfermedades cada vez más comunes en México. Ann hepatol. julio-

\section{(3): 282-8. ISNN 1665-2681}

Moreno T. (2015) La Diabetes mellitus en la cirrhosis hepatica. An. Fac. Cien. Med. Asunción) Vol. 48

(2) p.36-37. Recuperado de: http://dx doi.org/ 10.18004 /anales / 2015.048 (02) 33-040

Santos, L., Hernández, G, Varón Puerta A, Beltrán Ó, Botero, Rafael C, Mejía, G. (2010), Enfermedad

hepática por infiltración grasa no alcohólica. La nueva pandemia del milenio. Rev. Col. Gast, vol.

25, (4) oct-dic, pp. 380-398. Recuperado de http://redalycorg/articulo oa? id=337731599010

Tolman K, Fonseca V, Dalpiaz A, Tan M. (2007), Spectrum of liver disease in type 2 diabetes and management of patients with diabetes and liver disease. Diabetes Care, 30 pp. 734-743 http://dx.doi.org/10.2337/dc06-1539

Trombetta M, Spiazzi G, Zoppini G, Muggeo M: (2005) Review article: type 2 diabetes and chronic liver

disease in the Verona diabetes study. Aliment Pharmacol Ther 22 (Suppl. 2):24 -27, 2005. DOI: $10.1111 / \mathrm{j} .1365-2036.2005 .02590 . \mathrm{x}$

Wei M., Gibbons LW., Mitchell TL, Kampert JP, Blair SN (2000) Diabetes Care 2000 Jan; 23 (1): $18-22$ http://dx.doi.org/10.2337/diacare.23.1.18 
«Autor $1 », \ll$ Autor $2 », \ll$ Autor $3 » \ldots$

98 UNESUM-Ciencias. Publicación cuatrimestral. Vol. 3, Año 2019, No. 3 (Septiembre - Diciembre) 